\title{
Oculomotor Activity During Reading in Elementary School-Aged Students with Different Cognitive Skills
}

\author{
Ekaterina A. Budenkova* and Irina A. Vakoliuk \\ Immanuel Kant Baltic Federal University, School of Life Science \\ Kaliningrad, Russian Federation
}

Received 02.11.2018, received in revised form 13.07.2019, accepted 11.10.2019

\begin{abstract}
Eye movements provide relevant information about the functional state of some cognitive functions. The connection between eye movements and cognitive processes is based on the anatomical and functional overlap of structures controlling them in different parts of the brain. Various human activities, including reading, are associated with eye movements. Reading skills include two components: reading technique (a motor component) and reading comprehension (a cognitive component). Monitoring and interpretation of oculomotor response is a useful tool for studying the reading process. The present study characterizes saccadic and fixation eye-movement components in 7-8 year-old elementary school students that showed poor reading comprehension. The students were divided into three groups based on answer quality: giving no answer, giving a wrong answer, and coping with the task only with a prompt. The groups were found to differ in the amplitude, velocity, and deceleration of pro- and regressive saccades and the average duration of fixations per word. Thus, eye-movement parameters can be used to characterize the groups of schoolchildren that experience difficulty in reading comprehension or in formulating an answer to a semantic question. The present study contributes insight into various cognitive reading strategies and can be useful for creating personal learning paths for elementary school students to master reading skills, which are important for social success.
\end{abstract}

Keywords: eye tracking, saccades, gaze fixation, reading, elementary school-aged students.

Citation: Budenkova E.A., Vakoliuk I.A. Oculomotor activity during reading in elementary school-aged students with different cognitive skills. J. Sib. Fed. Univ. Biol., 2020, 13(3), 280-296. DOI: 10.17516/1997-1389-0328

(C) Siberian Federal University. All rights reserved

This work is licensed under a Creative Commons Attribution-NonCommercial 4.0 International License (CC BY-NC 4.0).

* Corresponding author E-mail address: KBudenkova@gmail.com

ORCID: 0000-0003-4854-5459 (Budenkova E.) 


\title{
Параметры движений глаз у младших школьников при оценке когнитивного компонента чтения
}

\author{
Е.А. Буденкова, И.А. Ваколюк \\ Балтийский федеральный университет \\ им. И. Канта, Институт живых систем \\ Российская Федерациия, Калининград
}

\begin{abstract}
Аннотация. Движения глаз могут отражать релевантную информацию о функциональном состоянии некоторых когнитивных функций. Взаимосвязь движений глаз и когнитивных процессов основана на анатомо-функциональном перекрытии контролирующих их структур в различных отделах головного мозга. С движениями глаз связаны многие формы активности человека, в том числе и чтение. Навык чтения складывается из двух компонентов: техника чтения (моторный компонент) и понимание читаемого текста (когнитивный компонент). Регистрация и интерпретация глазодвигательных реакций представляют собой полезный инструмент для изучения процессов чтения. В нашем исследовании получена характеристика саккадического и фиксационного компонентов движений глаз у школьников 7-8 лет с неудовлетворительным уровнем понимания читаемого текста, с выделением трех групп на основе качества ответа на вопрос (не давшие ответ, давшие неправильный ответ или справившиеся с задачей только после подсказки). Установлены особенности этих групп по амплитуде и скорости про- и регрессивных саккад, а также средней продолжительности фиксаций на слово. Исходя из полученных результатов, можно охарактеризовать группы учеников, испытавших сложность с пониманием смысла читаемого текста или формулировкой ответа на смысловой вопрос, через параметры движений глаз. Полученные результаты вносят вклад в понимание разных типов когнитивных стратегий чтения, что представляется полезным для построения индивидуальных траекторий обучения школьников начальных классов такому важному навыку, как чтение, необходимому для успешного социального функционирования.
\end{abstract}

Ключевые слова: айтрекинг, саккады, фиксации взора, чтение, младшие школьники.

Цитирование: Буденкова, Е.А. Параметры движений глаз у младших школьников при оценке когнитивного компонента чтения / Е.А. Буденкова, И.А. Ваколюк // Журн. Сиб. федер. ун-та. Биология, 2020. 13(3). С. $280-296$. DOI: $10.17516 / 1997-1389-0328$

\section{Введение}

Начиная со второй половины XIX в. вопрос развития эффективного навыка чтения приобрел широкий интерес, но, несмотря на многообразие разработанных стратегий чтения, проблема остается актуальной. Так, по данным PISA 2015 (Programme for International Student Assessment), среди 70 стран школьни- ки России в возрасте 15 лет занимают 26 место в области читательской грамотности. В качестве инструмента для оценки развития навыка чтения внимание исследователей привлекла окуломоторная активность (Дубасова, 2010; Clifton et al., 2016; Foster et al., 2018; Гарипова, 2018), базовыми компонентами которой являются саккады и фиксации. Сак- 
када - это краткий быстрый перевод взора с одного объекта на другой. Фиксация - это относительно неподвижная установка взора на объекте, состоящая из низкоамплитудных высокочастотных саккад (микросаккад). С помощью метода регистрации движений глаз (айтрекинг) создан ряд математических и теоретических моделей чтения с целью изучить механизм чтения и выявить его максимально эффективный, «идеальный», алгоритм (Дубасова, 2010).

Значительное количество работ посвящено изучению окуломоторной активности школьников с хорошо и плохо сформированным навыком чтения, при чтении текстов разной сложности и неоднозначности. Смена «технической» стратегии чтения на «информационную» после 6 лет под влиянием усиления лингвистических и семантических факторов и совершенствования когнитивных функций сопровождается сменой окуломоторной стратегии чтения, например, уменьшается количество просаккад (движения по направлению чтения) и регрессивных саккад (возвратных саккад). При сформированном навыке чтения продолжительность фиксаций и время ожидания саккады в среднем составляют 200-250 мс, продолжительность саккад 10-80 мс, а скорость саккад принимает значение 200-600 град/с (Дубасова, 2010; Seassau, Bucci, 2013).

Нейрональный субстрат глазодвигательных реакций тесно связан с обеспечением когнитивных функций (Kustov, Robinson, 1996; Hutton, 2008; Zhao et al., 2012; Eckstein et al., 2017), поэтому движения глаз представляют интерес в качестве нейрофизиологического маркера состояния внимания, памяти, восприятия, мышления и более сложного интегративного процесса, такого как чтение. Принято считать, что когнитивный компонент глазодвигательных реакций обе- спечивается корковыми взаимодействиями. Например, повышенная активность теменновисочной коры (РТС) связана со звукобуквенным декодированием, рост активности префронтальной коры (PFC) левого полушария и дорсолатеральной префронтальной коры (dlPFC) правого полушария - с рабочей памятью (Sharp et al., 2004). Длительность фиксаций связывается главным образом с трудностью идентификации слов в тексте (Безруких, Иванов, 2015) и коррелирует с увеличением активности в вентральной части височно-затылочной коры (vTOC) левого полушария (Steindorf, Rummel, 2020), которая отвечает за визуально-орфографическое распознавание слов по памяти (Rodrigues et al., 2019). Косвенный вклад в когнитивную регуляцию движений глаз привносят и стволовые структуры мозга (Voogd et al., 2012; Mäki-Marttunen et al., 2019; Schmahmann et al., 2019). Регуляция саккад и фиксаций осуществляется по общему принципу (Leigh, Zee, 2015). В регуляции саккад задействованы многие структуры головного мозга, сбои на любом из уровней могут отразиться на окулограмме (графическое отображение движений глаз) качественно и количественно. Сигнал к началу саккады генерируют пачечные нейроны (BNs) ретикулярной формации, стимулируемые глубинными слоями верхних бугорков четверохолмия (SC) среднего мозга, которые, в свою очередь, имеют большое количество прямых и непрямых связей с корой и подкорковыми образованиями (Leigh, Zee, 2015). Переход в режим фиксации, вероятно, связан с передачей сигнала от OMV (окуломоторная часть червя мозжечка) на $\mathrm{rSC}$ (ростральная часть $\mathrm{SC}$ ) и омнипаузные нейроны (OPNs) продолговатого мозга (Optican, Pretegiani, 2017).

Продолжительность фиксаций между саккадами отражает процессы интеграции и анализа входящей информации (Иванов и др., 
2010; Bezrukikh, Ivanov, 2013; Frey et al., 2013; Rayner, Reingold, 2015; Rucci, Poletti, 2015; Lopez-Perez et al., 2016). Во время фиксации происходит смещение внимания, селекция места следующей фиксации и запоминание выбранной локации, принимается решение о генерации коррекционной или регрессивной (возвратной) саккады (Leigh, Zee, 2015). Распределение фиксаций (пространственное и временное) и направление саккад служат критерием оценки зон интереса наблюдателя, распределения внимания. При выполнении саккад также происходит обработка поступающей информации: восприятие и распознавание слов, транссаккадическое запоминание, семантический и идентификационный прайминг (ускоренное опознание слова, когда ему предшествовало слово, связанное с ним по смыслу) и др. (Irwin, Thomas, 2007; Schneegans et al., 2014; Yeh et al., 2014; Edwards et al., 2018). Особый интерес представляет процесс транссаккадического запоминания, связанный с переносом информации между пре- и постсаккадическим этапом обработки информации (Stewart, Schütz, 2018). Транссакадическое запоминание на всех этапах зависит от уровня внимания. Помимо этого, известно, что динамика диаметра зрачка, кроме реакции на свет, коррелирует с общим уровнем бодрствования, внимания и рабочей памяти за счет проекций от голубого пятна (Aminihajibashi et al., 2019; Hakim et al., 2019).

С учетом многокомпонентности и индивидуальности когнитивной регуляции чтения представляется интересным более подробное разделение и описание группы плохо понимающих читаемый текст учеников. В связи с этим целью данного исследования является характеристика глазодвигательных реакций учеников младшего школьного возраста, имеющих разные уровни понимания текста при чтении.

\section{Материалы и методы}

В исследовании приняли участие 117 человек (63 мальчика, 54 девочки), ученики вторых классов МБОУ СОШ «Школа будущего» г. Калининграда (средний возраст 8 лет $2 \pm$ 6 мес.). Все участники исследования - здоровые дети с нормальным развитием. Перед проведением исследования родители подписали информированное согласие на участие детей в исследовании. Исследование проводили с соблюдением условия экологической валидности. Проведение исследования было одобрено локальным российским этическим комитетом города Санкт-Петербурга, чья деятельность согласуется с Хельсинской Декларацией, WHO Guidelines, ICH GCP и законами РФ.

У учеников зарегистрировали движения глаз в процессе чтения с помощью аппарата RED250mobile (SensoMotoric Instruments, www.smivision.com). Данная система соответствует европейским стандартам медицинского оборудования (CE-marked, FCC EyeSafety). REDm независимо регистрирует движение обоих глаз. Частота регистрации $120 \mathrm{~Hz}$, разрешающая способность 0,1 углового градуса. RED-m обладает функцией автоматической компенсации движений головы испытуемого. Система обладает достаточной гибкостью и подходит для людей разного возраста и зрения с учетом таких параметров, как цвет глаз, наличие очков и линз.

Во время выполнения заданий испытуемый сидел напротив монитора лэптопа на расстоянии $60 \pm 5 \mathrm{~cm}$. Тестирование одного испытуемого занимало около 5 мин. Перед началом выполнения задания для каждого испытуемого проводилась калибровка аппарата, испытуемого инструктировали: сфокусировать внимание на центре калибровочной точки, которая движется на экране. После того как аппарат проходил калибровку, на 
экране появлялся отрывок текста, испытуемым предлагалось прочесть его вслух, при этом отмечалось количество ошибок при чтении (замена букв/слов, орфоэпические ошибки учитывались в общем), тип чтения (по слогам, по словам, бегло) и время чтения. После прочтения текста ученику задавали вопрос о содержании прочитанного («Как думаешь, о чем ты прочел(а) в тексте?», правильный ответ - черепаха). Стимульный материал отрывок текста из учебника для вторых классов (55 слов, текст без синтаксической неоднозначности), шрифт Times New Roman, 14 кегль:

“Это животное часто заводят в живом уголке в школе и дома, так как оно достаточно неприхотливо. Лежит себе в коробочке или аквариуме, ест мало, ничего не просит. Туловище этого животного покрыто панцирем, состоящим из отдельных щитков, а ноги, голова и хвост - роговыми чешуйками. Животное относится к хладнокровным, его поведение полностью зависит от температуры окружающей среды.”

Численные параметры (амплитуда, продолжительность, средняя и пиковая скорость саккад, длительность фиксаций на слово) были получены с помощью программы BeGaze 3.0 (SMI, Германия). Для характеристики просаккад (по направлению чтения), регрессивных саккад (против направления текста), саккад, переводящих взор на начало новой строки, проведена оценка следующих параметров: длительность и амплитуда, средняя и пиковая скорость, замедление, количество саккад и морганий. Для оценки фиксационной составляющей выбраны следующие параметры: продолжительность фиксаций после просаккад, регрессивных саккад и перевода взгляда на новую строку, динамика диаметра зрачка. В результатах описаны только те параметры, по отношению к которым были обнаружены статистически значимые внутри- или/и межгрупповые различия.

Статистическую обработку полученного материала проводили с использованием SPSS 21.0. Учитывая гетерогенность по количественному составу групп и нормальности распределения, приняли решение применить непараметрические критерии для статистического анализа. Для проверки нормальности распределения параметров использовали критерий Колмогорова-Смирнова (k-s - стандартизированное значение критерия, при $\mathrm{p} \leq 0,05$ принимается гипотеза о нормальности распределения значений параметра). Обнаружено, что в группах часть параметров имеет нормальное распределение и часть ненормальное. Учитывая это, а также малый объем выборки в группах NoA и RwP, для статистической обработки данных выбрали непараметрические критерии. Для сравнения двух несвязанных выборок использовали непараметрический U-критерий Манна-Уитни с поправкой Бонферрони на множественность сравнений (U - критерий Манна-Уитни, Z показатель отклонения средних значений по выборкам в пределах трех сигм U-критерия, наличие различий при $p \leq 0,01)$, для сравнения двух связанных выборок - непараметрический $\mathrm{W}$-критерий Вилкоксона с поправкой Бонферрони на множественность сравнений ( $\mathrm{W}$ - значение критерия Вилкоксона, наличие различий при $p \leq 0,016$ ).

\section{Результаты}

Ученики были разделены на группы по степени понимания прочитанного текста (табл. 1), критерием служил ответ на вопрос о его содержании. Выделены ученики, правильно ответившие на вопрос (группа Right или $\mathrm{R}$ ), неправильно ответившие на вопрос (примеры ответов: подводная лодка, фильтр, рыбы и др.) и не давшие никакого ответа. 
Таблица 1. Характеристика участников

Table 1. Sample characteristics

\begin{tabular}{|c|c|c|c|c|c|c|c|c|c|}
\hline \multirow{3}{*}{ 蛋 } & \multirow{3}{*}{ 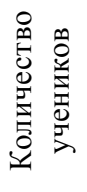 } & \multirow{3}{*}{ 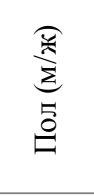 } & \multirow{3}{*}{ 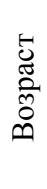 } & \multicolumn{6}{|c|}{ Тип чтения } \\
\hline & & & & \multicolumn{2}{|c|}{ По слогам } & \multicolumn{2}{|c|}{ По словам } & \multicolumn{2}{|c|}{ Бегло } \\
\hline & & & & $\begin{array}{l}\text { Кол-во } \\
\text { чел., \% }\end{array}$ & $\begin{array}{c}\mathrm{N} \\
\text { ошибок }\end{array}$ & $\begin{array}{l}\text { Кол-во } \\
\text { чел., \% }\end{array}$ & $\begin{array}{c}\mathrm{N} \\
\text { ошибок }\end{array}$ & $\begin{array}{l}\text { Кол-во } \\
\text { чел., \% }\end{array}$ & $\begin{array}{c}\mathrm{N} \\
\text { ошибок }\end{array}$ \\
\hline $\mathrm{R}$ & 59 & $28 / 25$ & \multirow{4}{*}{$\begin{array}{c}\vec{D} \\
\stackrel{D}{ } \\
\infty \\
1 \\
1\end{array}$} & 11 & $2-4$ & 5 & $1-5$ & 84 & 0 \\
\hline W & 32 & $28 / 29$ & & 14 & $2-4$ & 2 & $2-5$ & 84 & 0 \\
\hline NoA & 19 & $15 / 4$ & & 8 & $0-1$ & 1 & $0-1$ & 91 & 0 \\
\hline RwP & 7 & $1 / 6$ & & 23 & $1-3$ & 4 & $2-8$ & 73 & 0 \\
\hline
\end{tabular}

$\mathrm{R}$ - школьники, давшие правильный ответ на вопрос; $\mathrm{W}$ - школьники, давшие неправильный ответ на вопрос; NoA школьники, не давшие ответ на вопрос; RwP - школьники, давшие правильный ответ на вопрос после наводящих вопросов.

Всем ученикам, давшим неверный ответ или не ответившим, были заданы наводящие вопросы по содержанию текста. Ученики, которые смогли правильно ответить после наводящих вопросов («Это живое или неживое? Умеет ли это плавать?»), составили группу RwP (Right with prompting), те, которым не помогли наводящие вопросы, составили группы W (Wrong) и NoA (No answer). $\mathrm{B}$ каждой группе присутствуют дети с разными типами чтения и, соответственно, с разной скоростью чтения в сравнительно равных соотношениях (табл. 1).

Примеры окулограмм представлены на рис. 1 и 2, а численные значения окуломоторных параметров сведены в табл. 2. Статистически значимых различий по общему количеству на читаемый текст просаккад, регрессивных и межстрочных саккад (и, соответственно, фиксаций) между группами не обнаружено. Согласно литературным данным, регрессивные саккады в норме составляют 10-15 \% от общего числа саккадических движений (Smadja et al., 2019). В данном исследовании доля регрессивных саккад составила $13 \pm 7 \%$ вне зависимости от группы. Пиковая и средняя скорости саккад имеют схожий характер различий между группами, поэтому для удобства в дальнейшем будем описывать только среднюю скорость саккад.

Средние значения скорости и амплитуды саккад отображены на рис. 3 и 4 соответственно. Во всех группах средняя скорость просаккад и регрессивных саккад ниже, чем средняя скорость саккад при переводе взора на новую строку, что предсказуемо за счет исходно большей амплитуды межстрочных саккад (табл. 2). Внутригрупповое сравнение показало, что скорость и амплитуда регрессивных саккад достоверно выше, чем скорость и амплитуда просаккад в группах $\mathrm{R}$ и NoA (табл. 2, 3). Среднее значение скорости и амплитуды саккад не имеет статистически значимых различий между группами, но стоит отметить увеличение размаха значений скорости в группах W и NoA и размаха значений амплитуды в группах NoA и RwP.

На рис. 5 и 6 приведены, соответственно, средние значения продолжительности фиксаций после саккад и диаметра зрачка при фиксациях. Средняя продолжительность фиксации на слово после просаккад и после перехода на новую строку статистически значимо выше в группе RwP по сравнению с остальными группами (табл. 4). Средняя продолжительность фиксаций после регрессив- 

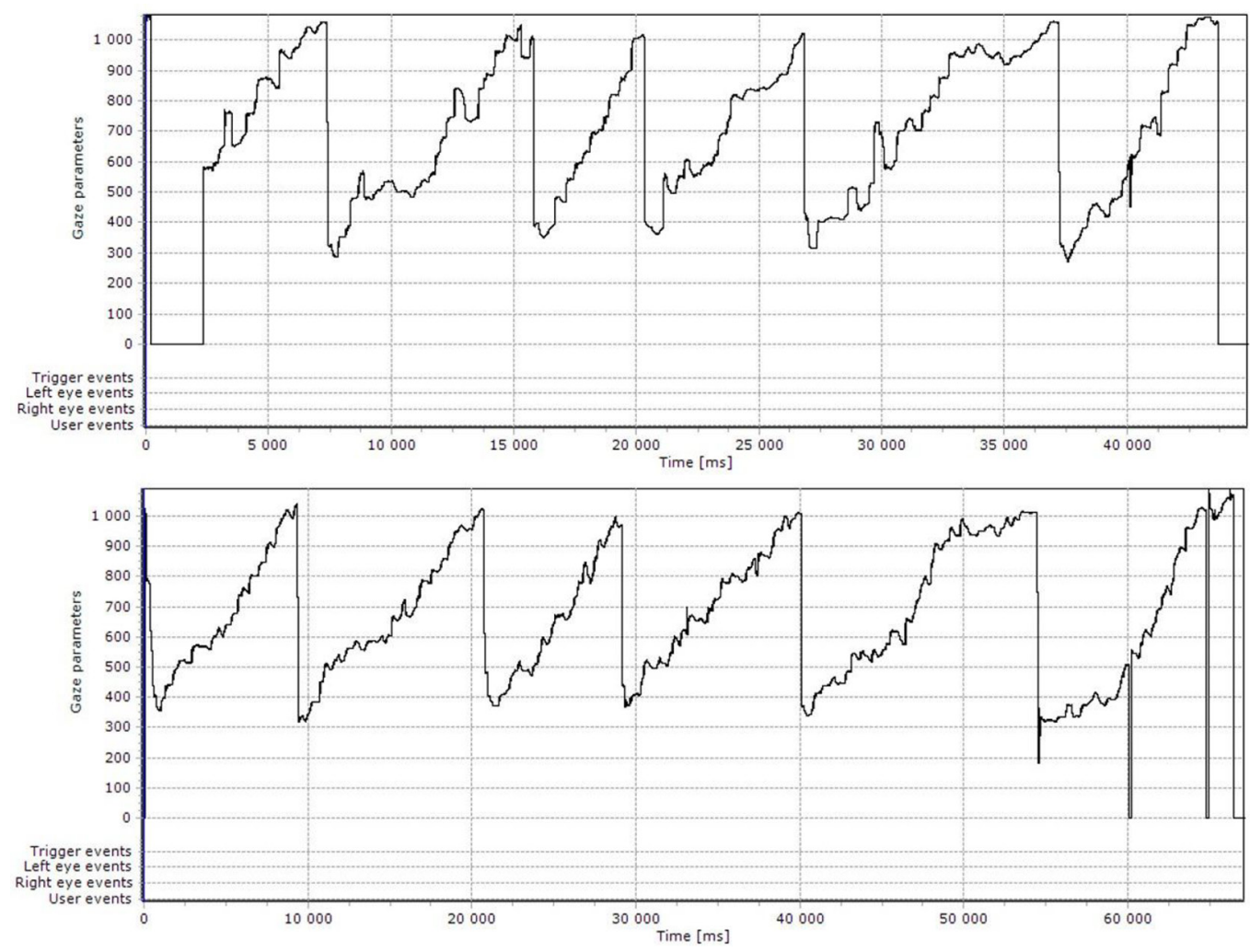

Рис. 1. Примеры окулограмм при чтении: сверху - ученик К., правильно ответивший на вопрос после чтения (группа R); снизу - ученик А., неправильно ответивший на вопрос после чтения (группа W)

Fig. 1. Examples of schoolchildren's video-oculograms recorded during reading: top - school student K., who gave the correct answer to the question after reading (group R); bottom - school student A., who gave a wrong answer to the question after reading (group W)

ных саккад в группах R и W выше, чем в NoA и RwP (табл. 4). При этом продолжительность фиксаций после регрессивных саккад внутри группы W выше, чем после просаккад и перехода на новую строку (табл. 3). А в группе RwP продолжительность фиксации после регрессивных саккад меньше, чем после просаккад и перехода на новую строку (табл. 3). Среднее значение диаметра зрачка при фиксациях после саккад в группах $\mathrm{R}$ и $\mathrm{W}$ статистически значимо ниже, чем в NoA и RwP (табл. 4, рис. 6). Внутригрупповое сравнение показало, что в группе R диаметр зрачка при фиксациях после регрессивных саккад выше, чем при фиксациях после просаккад и после перехода на новую строку (табл. 3).

\section{Обсуждение}

Цель данного исследования заключалась в выявлении особенностей окуломоторной активности у школьников 7-8 лет с разным уровнем вдумчивости чтения. В отличие от работ других авторов, в данном исследовании не было обнаружено зависимости между количеством фиксаций/саккад, скоростью чтения и степенью понимания текста (Безруких, Иванов, 2015; Vorstius et al., 2014; Steindorf, Rummel, 2020). Скорость чтения и количество фиксаций/саккад естественным образом зависят от типа чтения, однако распределение участников в группы по уровню понимания текста показало, что в каждой группе присутствуют ученики с разным типом чтения (табл. 1). 

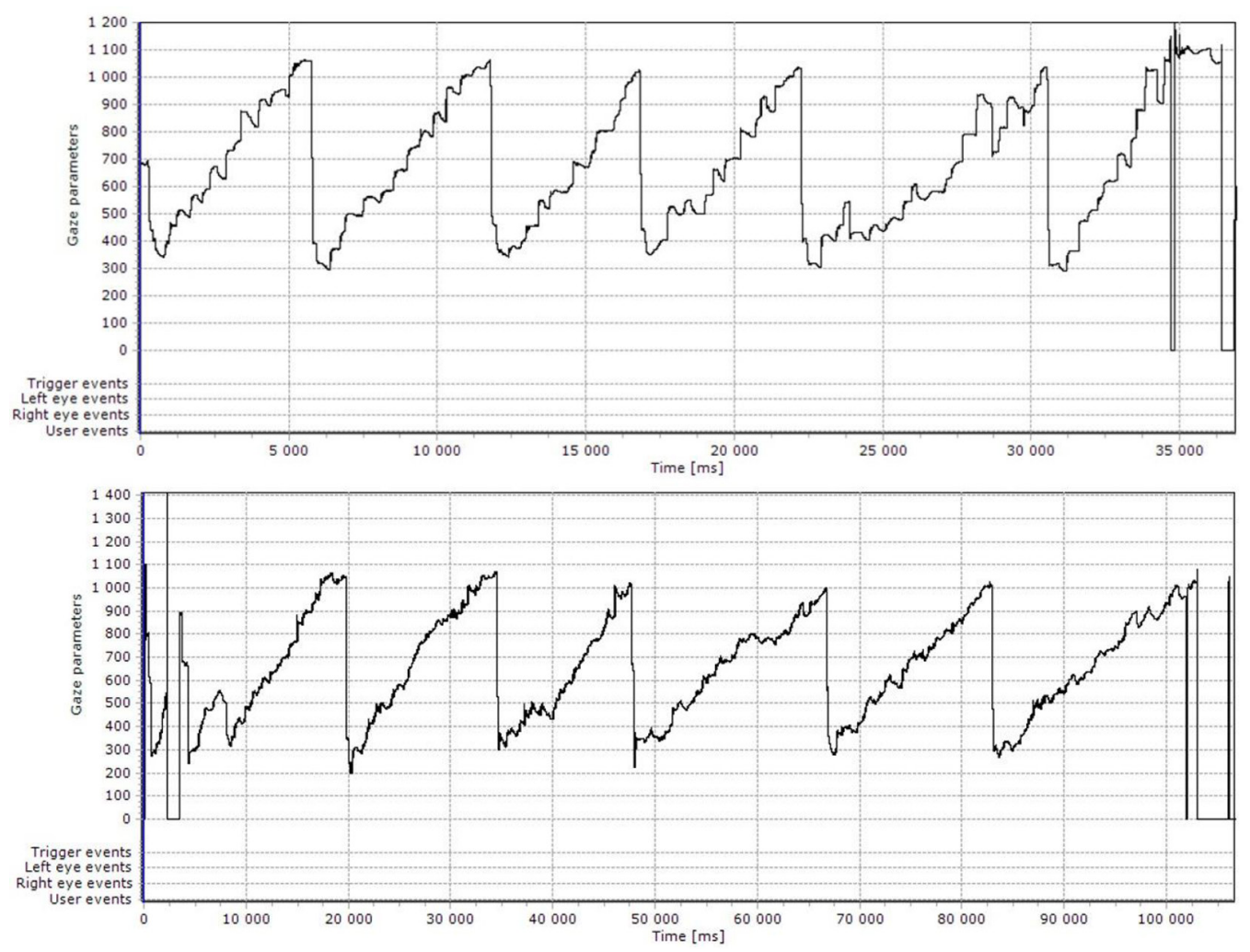

Рис. 2. Примеры окулограмм при чтении: сверху - ученик Т., не ответивший на вопрос после чтения (группа NoA); снизу - ученик Д., правильно ответивший на вопрос после чтения, но после наводящих вопросов (группа RwP)

Fig. 2. Examples of schoolchildren's video-oculograms recorded during reading: top - school student T., who did not give any answer (group NoA); bottom - school student D., who gave the correct answer to the question with a prompt (group RwP)

Таблица 2. Значения скорости и амплитуды саккад, продолжительности фиксаций и диаметр зрачка для школьников всех групп

Table 2. Saccadic velocity, saccadic amplitude, duration of fixation and pupil diameter for all groups

\begin{tabular}{|c|c|c|c|c|c|c|}
\hline \multirow[b]{2}{*}{ Группы } & \multicolumn{2}{|c|}{ Просаккады } & \multicolumn{2}{|c|}{ Регрессивные саккады } & \multicolumn{2}{|c|}{ Межстрочные саккады } \\
\hline & $\begin{array}{c}\text { медиана; } \\
\text { CI }\end{array}$ & $\begin{array}{c}\text { k-s; } \\
\text { p-value }\end{array}$ & $\begin{array}{c}\text { медиана; } \\
\text { CI }\end{array}$ & $\begin{array}{c}\text { k-s; } \\
\text { p-value }\end{array}$ & $\begin{array}{c}\text { медиана; } \\
\text { CI }\end{array}$ & $\begin{array}{c}\text { k-s; } \\
\text { p-value }\end{array}$ \\
\hline 1 & 2 & 3 & 4 & 5 & 6 & 7 \\
\hline \multicolumn{7}{|c|}{ Скорость саккад при чтении, градус/с } \\
\hline $\mathrm{R}$ & 24,$9 ; 6,3$ & 1,$5 ;<0,05$ & 46,$0 ; 12,4$ & 0,$4 ;>0,05$ & 168,$0 ; 10,8$ & 1,$2 ;>0,05$ \\
\hline $\mathrm{W}$ & 21,$1 ; 7,1$ & 0,$5 ;>0,05$ & 28,$1 ; 11,7$ & 0,$9 ;>0,05$ & 154,$6 ; 16,2$ & 1,$2 ;<0,05$ \\
\hline NoA & 14,$4 ; 8,2$ & 1,$9 ;<0,05$ & 41,$4 ; 15,1$ & 1,$3 ;>0,05$ & 150,$3 ; 24,0$ & 1,$4 ;<0,05$ \\
\hline RwP & 15,$2 ; 13,5$ & 0,$8 ;>0,05$ & 19,$8 ; 15,4$ & 1,$1 ;<0,05$ & 148,$5 ; 12,1$ & 1,$0 ;<0,05$ \\
\hline \multicolumn{7}{|c|}{ Амплитуда саккад при чтении, градус } \\
\hline $\mathrm{R}$ & 0,$4 ; 0,02$ & 1,$4 ;<0,05$ & 0,$8 ; 0,5$ & 0,$7 ;>0,05$ & 12,$4 ; 1,2$ & 1,$4 ;<0,05$ \\
\hline W & 0,$3 ; 0,1$ & 1,$0 ;>0,05$ & 0,$5 ; 0,2$ & 0,$9 ;>0,05$ & 11,$0 ; 0,8$ & 1,$6 ;<0,05$ \\
\hline
\end{tabular}


Продолжение табл. 2

Continued Table 2

\begin{tabular}{|ccccccc|}
\hline 1 & 2 & 3 & 4 & 5 & 6 & 7 \\
\hline NoA & 0,$2 ; 0,01$ & 2,$4 ;<0,05$ & 0,$7 ; 0,3$ & 1,$2 ;>0,05$ & 11,$0 ; 3,2$ & 0,$6 ;>0,05$ \\
RwP & 0,$3 ; 0,2$ & 1,$1 ;>0,05$ & 0,$3 ; 0,2$ & 1,$7 ;<0,05$ & 11,$0 ; 2,2$ & 1,$3 ;<0,05$ \\
\hline \multicolumn{7}{c}{ Длительность фиксаций при чтении, мс } \\
\hline R & 683,$8 ; 83,5$ & 2,$0 ;<0,05$ & 992,$3 ; 86,7$ & 4,$5 ;<0,05$ & 672,$3 ; 32,0$ & 2,$9 ;<0,05$ \\
W & 692,$3 ; 68,2$ & 1,$3 ;<0,05$ & $1106 ; 117$ & 1,$7 ;<0,05$ & 884,$2 ; 42,7$ & 2,$0 ;<0,05$ \\
NoA & 508,$1 ; 54,5$ & 1,$4 ;<0,05$ & 615,$7 ; 69,8$ & 4,$6 ;<0,05$ & 523,$8 ; 25,0$ & 2,$2 ;<0,05$ \\
RwP & $1123 ; 85$ & 1,$1 ;>0,05$ & 674,$1 ; 62,5$ & 3,$0 ;<0,05$ & 2672,$1 ; 28,0$ & 3,$5 ;<0,05$ \\
\hline & \multicolumn{7}{c}{ Диаметр зрачка при фиксациях, мм } & & 2,$07 ;<0,05$ \\
R & 4,$20 ; 0,03$ & 2,$10 ;<0,05$ & 4,$30 ; 0,02$ & 1,$20 ;<0,05$ & 4,$10 ; 0,03$ & 2,03 \\
W & 4,$20 ; 0,03$ & 1,$79 ;<0,05$ & 4,$30 ; 0,03$ & 2,$50 ;<0,05$ & 4,$20 ; 0,03$ & 2,$50 ;<0,05$ \\
NoA & 4,$60 ; 0,03$ & 1,$50 ;<0,05$ & 4,$60 ; 0,01$ & 4,$80 ;<0,05$ & 4,$60 ; 0,03$ & 1,$50 ;>0,05$ \\
RwP & 4,$70 ; 0,05$ & 1,$20 ;>0,05$ & 4,$80 ; 0,10$ & 0,$80 ;>0,05$ & 4,$70 ; 0,03$ & 4,$10 ;>0,05$ \\
\hline
\end{tabular}

Обозначения групп как в табл. 1; CI - доверительный интервал; k-s - значение критерия Колмогорова-Смирнова, при $\mathrm{p} \leq 0,05$ принимается гипотеза о нормальности распределения значений.

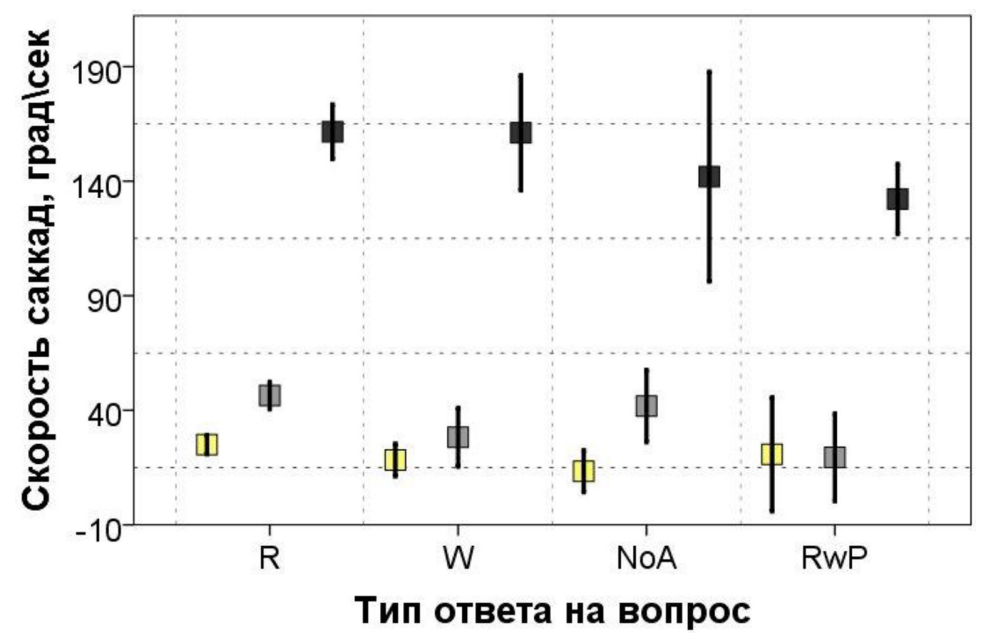

Рис. 3. Среднее значение (медиана) скорости саккад при чтении, столбики ошибок обозначают 95\%-ный доверительный интервал: столбики с желтым маркером - фиксации после просаккад, столбики с серым маркером - фиксации после регрессивных саккад, столбики с черным маркером - фиксации после перехода на новую строку; тип ответа на вопрос: правильный ответ (группа R), неправильный ответ (группа W), без ответа (группа NoA), правильный ответ после подсказки (группа RwP)

Fig. 3. Median saccadic velocity during reading, the $95 \%$ confidence interval: yellow marker - prosaccades, gray marker - regressive saccades, black marker - interline saccades; type of answer to question: right answer (group R), wrong answer (group W), no answer (group NoA), right only with a prompt (group RwP) 


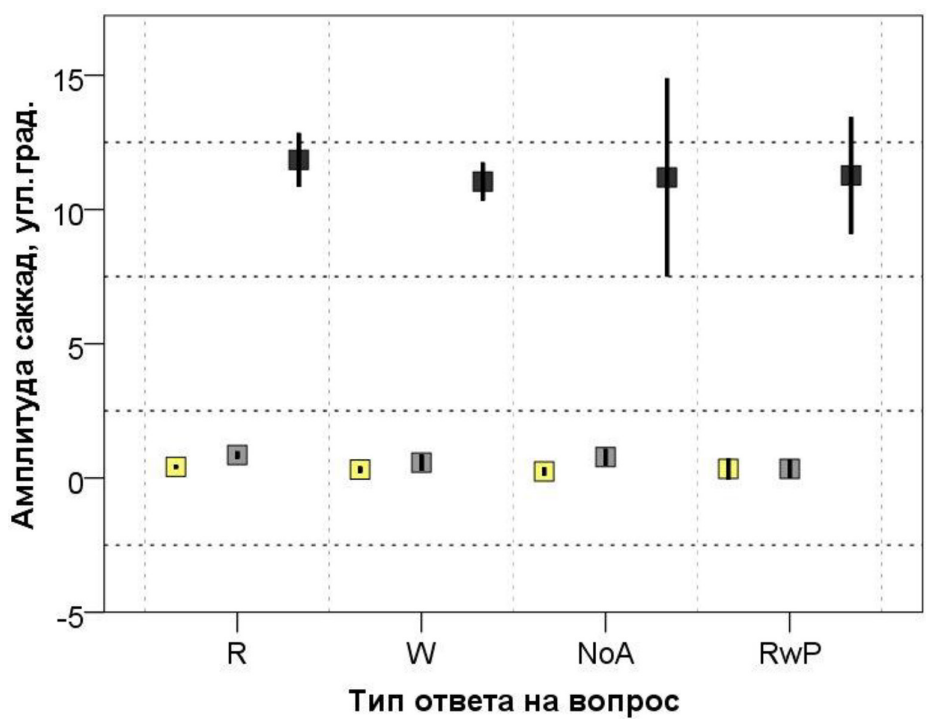

Рис. 4. Средняя (медиана) амплитуда саккад при чтении, столбики ошибок обозначают 95\%-ный доверительный интервал: столбики с желтым маркером - фиксации после просаккад, столбики с серым маркером - фиксации после регрессивных саккад, столбики с черным маркером - фиксации после перехода на новую строку; типы ответа на вопрос - см. рис. 3

Fig. 4. Median saccadic amplitude during reading, the 95\% confidence interval: yellow marker - prosaccades, gray marker - regressive saccades, black marker - interline saccades; type of answer to question - see Fig. 3

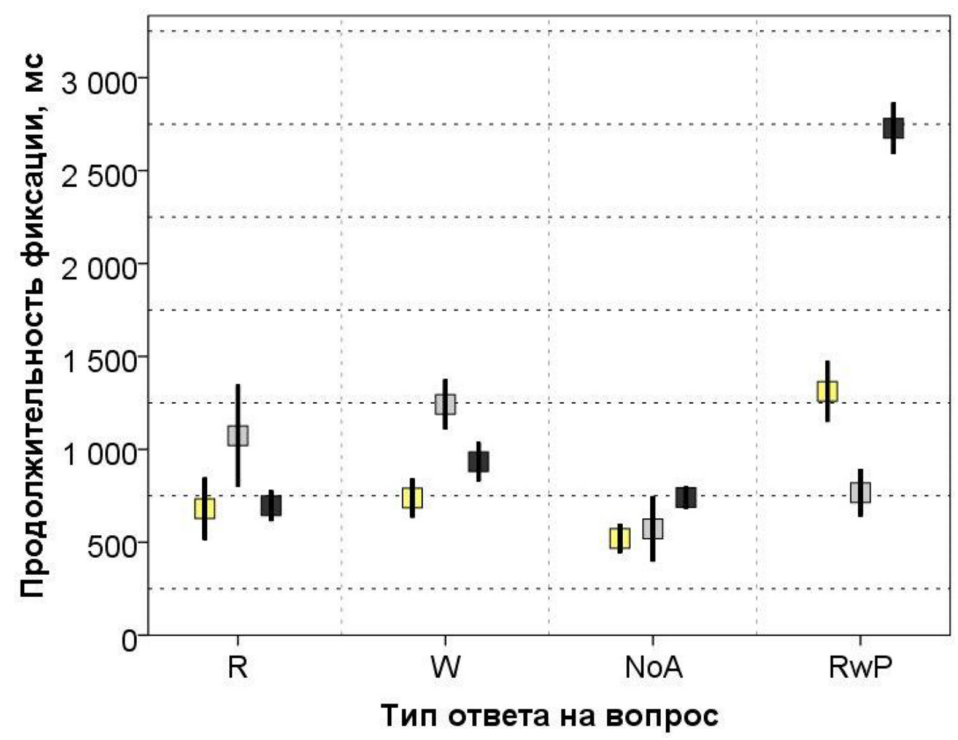

Рис. 5. Средняя (медиана) длительность фиксации на слово, столбики ошибок обозначают 95\%-ный доверительный интервал: столбики с желтым маркером - фиксации после просаккад, столбики с серым маркером - фиксации после регрессивных саккад, столбики с черным маркером - фиксации после перехода на новую строку; типы ответа на вопрос - см. рис. 3

Fig. 5. Median fixation duration, the 95\% confidence interval: yellow marker - prosaccades, gray marker regressive saccades, black marker - interline saccades; type of answer to question - see Fig. 3 
Таблица 3. Внутригрупповое сравнение окуломоторных параметров

Table 3. Intragroup comparison of oculomotor parameters

\begin{tabular}{|c|c|c|c|c|c|c|c|c|c|c|c|c|}
\hline & \multicolumn{12}{|c|}{ Скорость саккад } \\
\hline \multirow{3}{*}{ 兽 } & $\mathbf{R}$ & reg & int & $\mathbf{W}$ & reg & int & NoA & reg & int & RwP & reg & int \\
\hline & pro & $\begin{array}{l}\mathrm{W}=43 \\
\mathrm{p}<0,01\end{array}$ & - & pro & - & - & pro & $\begin{array}{l}\mathrm{W}=56 \\
\mathrm{p}<0,01\end{array}$ & - & pro & - & - \\
\hline & int & - & & int & - & & int & - & & int & - & \\
\hline & \multicolumn{12}{|c|}{ Амплитуда саккад } \\
\hline \multirow{4}{*}{$\begin{array}{l}\text { 思 } \\
\text { 总 }\end{array}$} & $\mathbf{R}$ & reg & int & $\mathbf{W}$ & reg & int & NoA & reg & int & RwP & reg & int \\
\hline & pro & $\begin{array}{l}\mathrm{W}=42 \\
\mathrm{p}<0,01\end{array}$ & - & pro & - & - & pro & $\begin{array}{l}\mathrm{W}=56 \\
\mathrm{p}<0,01\end{array}$ & - & pro & - & - \\
\hline & int & - & & int & - & & int & - & & int & - & \\
\hline & \multicolumn{12}{|c|}{ Продолжительность фиксаций } \\
\hline \multirow{4}{*}{ 昤 } & $\mathbf{R}$ & reg & int & $\mathbf{W}$ & reg & int & NoA & reg & int & RwP & reg & int \\
\hline & pro & - & - & pro & $\begin{array}{l}W=75 \\
p<0,01\end{array}$ & - & pro & - & - & pro & $\begin{array}{c}\mathrm{W}=30 \\
\mathrm{p}<0,01\end{array}$ & - \\
\hline & int & - & & int & $\begin{array}{l}W=34 \\
p<0,01\end{array}$ & & int & - & & int & $\begin{array}{c}\mathrm{W}=99 \\
\mathrm{p}<0,01\end{array}$ & \\
\hline & \multicolumn{12}{|c|}{ Диаметр зрачка } \\
\hline \multirow{3}{*}{$\begin{array}{l}\text { 息 } \\
\text { 忩 }\end{array}$} & $\mathbf{R}$ & reg & int & $\mathbf{W}$ & reg & int & NoA & reg & int & RwP & reg & int \\
\hline & pro & $\begin{array}{l}W=76 \\
p<0,01\end{array}$ & - & pro & - & - & pro & - & - & pro & - & - \\
\hline & int & $\begin{array}{c}\mathrm{W}=9 \\
\mathrm{p}<0,01\end{array}$ & & int & - & & int & - & & int & - & \\
\hline
\end{tabular}

Обозначения групп - см. табл. 1; pro - просаккады, reg - регрессивные саккады, int - межстрочные саккады; W значение W-критерия Вилкоксона, p - p-value с поправкой Бонферрони (статистически значимое различие при $\mathrm{p} \leq 0,016)$, прочерк обозначает отсутствие статистически значимых различий между сравниваемыми параметрами групп.

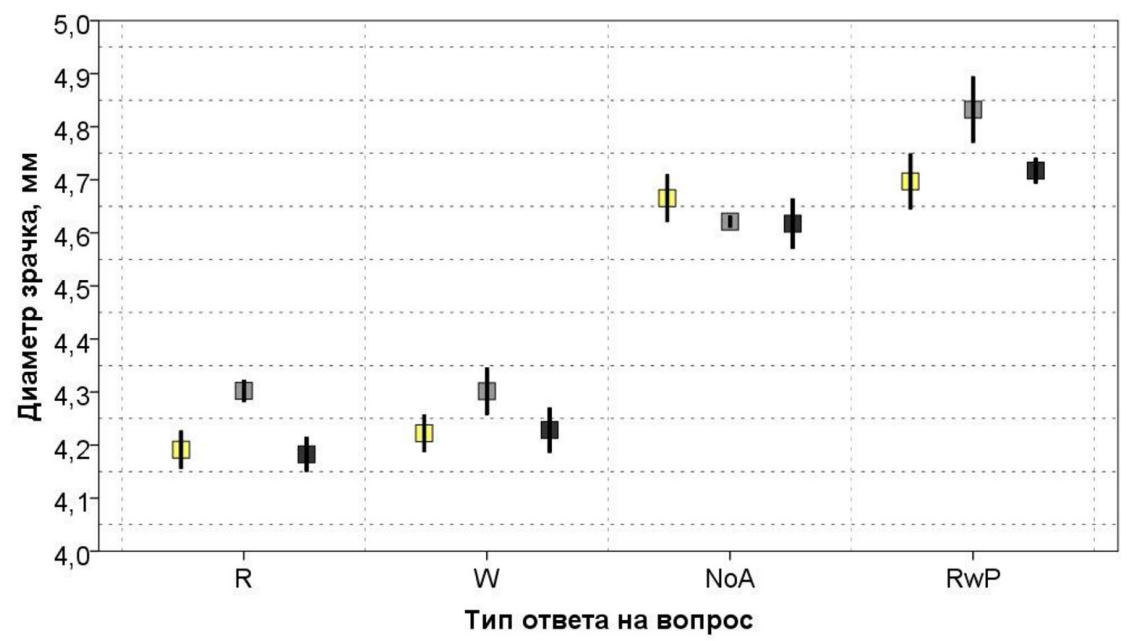

Рис. 6. Среднее значение (медиана) диаметра зрачка во время фиксаций при чтении, столбики ошибок обозначают 95\%-ный доверительный интервал: столбики с желтым маркером - фиксации после просаккад, столбики с серым маркером - фиксации после регрессивных саккад, столбики с черным маркером - фиксации после перехода на новую строку; типы ответа на вопрос - см. рис. 3

Fig. 6. Mean pupil diameter (median) during fixating, the $95 \%$ confidence interval: yellow marker - prosaccades, gray marker - regressive saccades, black marker - interline saccades; type of answer to question - see Fig. 3 
Таблица 4. Межгрупповое сравнение окуломоторных параметров

Table 4. Intergroup comparison of oculomotor parameters

\begin{tabular}{|c|c|c|c|c|c|c|c|c|c|c|c|c|c|c|c|c|c|c|}
\hline & \multicolumn{9}{|c|}{ Продолжительность фиксаций } & \multicolumn{9}{|c|}{ Диаметр зрачка при фиксациях } \\
\hline & $\mathrm{U}$ & z & $\mathrm{p}$ & $\mathrm{U}$ & $\mathrm{z}$ & $\mathrm{p}$ & $\mathrm{U}$ & $\mathrm{z}$ & $\mathrm{p}$ & $\mathrm{U}$ & z & $\mathrm{p}$ & $\mathrm{U}$ & $\mathrm{z}$ & $\mathrm{p}$ & $\mathrm{U}$ & $\mathrm{z}$ & $\mathrm{p}$ \\
\hline группы & \multicolumn{3}{|c|}{$\mathrm{R}$} & \multicolumn{3}{|c|}{$\mathrm{W}$} & \multicolumn{3}{|c|}{ NoA } & \multicolumn{3}{|c|}{$\mathrm{R}$} & \multicolumn{3}{|c|}{$\mathrm{W}$} & \multicolumn{3}{|c|}{ NoA } \\
\hline \multicolumn{19}{|c|}{ Просаккады } \\
\hline W & - & - & - & & & & & & & - & - & - & & & & & & \\
\hline NoA & - & - & - & - & - & - & & & & 79 & $-8,4$ & $<0,01^{*}$ & 99 & $-9,1$ & $<0,01^{*}$ & & & \\
\hline RwP & 21 & $-5,3$ & $<0,01^{*}$ & 19 & $-4,9$ & $<0,01^{*}$ & 40 & -7 & $<0,01 *$ & 66 & $-9,0$ & $<0,01^{*}$ & 100 & $-9,7$ & $<0,01^{*}$ & - & - & - \\
\hline \multicolumn{19}{|c|}{ Регрессивные саккады } \\
\hline W & - & - & - & & & & & & & - & - & - & & & & & & \\
\hline NoA & 29 & $-5,9$ & $<0,01 *$ & 23 & $-6,7$ & $<0,01^{*}$ & & & & 35 & $-6,8$ & $<0,01^{*}$ & 119 & $-9,6$ & $<0,01^{*}$ & & & \\
\hline RwP & 6 & $-2,7$ & $>0,01$ & 17 & $-5,2$ & $<0,01^{*}$ & - & - & - & 10 & $-8,9$ & $<0.01^{*}$ & 109 & $-8,5$ & $<0,01^{*}$ & - & - & - \\
\hline & \multicolumn{18}{|c|}{ Межстрочные саккады } \\
\hline W & - & - & - & & & & & & & - & - & - & & & & & & \\
\hline NoA & - & - & - & - & - & - & & & & 65 & $-8,5$ & $<0,01^{*}$ & 83 & $-8,8$ & $<0,01^{*}$ & & & \\
\hline RwP & 56 & $-9,7$ & $<0,01^{*}$ & 112 & $-10,0$ & $<0,01^{*}$ & 61 & -10 & $<0,01^{*}$ & 56 & $-10,1$ & $<0,01^{*}$ & 112 & $-11,3$ & $<0,01^{*}$ & - & - & - \\
\hline
\end{tabular}

Обозначения групп - см. табл. 1; U - значение критерия Манна-Уитни, z - стандартизированное значение критерия Манна-Уитни, p - p-value, * - поправка Бонферрони (статистически значимое различие при $\mathrm{p} \leq 0,01$ ), прочерк обозначает отсутствие статистически значимых различий между сравниваемыми параметрами групп. 
К основным полученным результатам можно отнести следующее: 1) средняя длительность фиксаций после просаккад и перехода на новую строку больше в группе RwP (наводящие вопросы экспериментатора приводят к инсайту), чем в остальных группах; 2) внутри группы W (неправильный ответ на вопрос) средняя длительность фиксаций после регрессивных саккад больше, чем после просаккад и перехода на новую строку; 3) внутри групп NoA (нет ответа) и R (правильный ответ) средняя скорость регрессивных саккад выше скорости просаккад; 4) в группах W, NoA и RwP увеличен размах средних значений скорости и амплитуды регрессивных саккад по сравнению с группой R; 5) среднее значение диаметра зрачка при фиксациях выше в группах NoA и $\mathrm{RwP}$ в сравнении с группами W и R.

Зарегистрированное в исследовании увеличение диаметра зрачка в NoA и RwP может отражать недостаточность тормозной норадреналинергической стимуляции сфинктера зрачка со стороны голубого пятна (Locus Coeruleus, LC). Известно, что помимо влияния на частоту сердечных сокращений, кровяное давление и состояние бодрствования, активность LC косвенно связана с регуляцией памяти (эпизодической и рабочей) и внимания за счет широкой сети проекций в головном мозге (Mather, Harley, 2016). Также диаметр зрачка может служить относительным показателем уровня тревожности (Keil et al., 2018). В группах NoA и RwP диаметр зрачка выше, чем в группах $\mathrm{R}$ и $\mathrm{W}$, из чего можно предположить, что в группах NoA и RwP наблюдается дефицит функций внимания и памяти, базовый или в ответ на когнитивную нагрузку.

Несмотря на то что чтение как сложный процесс зависит от целого ряда когнитивных умений и степени развития высших психических функций, результирующее понимание текста может определяться дефицитом какойлибо одной (или нескольких) когнитивной функции, наподобие «слабого звена». Так, в группе NoA навык чтения технически развит хорошо, т.е. преобладает беглый тип чтения без ошибок или с 1-2 ошибками, но целостного восприятия текста нет (нет ответа на смысловой вопрос). В литературе такой тип чтения принято называть механическим. Отличительной окуломоторной особенностью данной группы является увеличение размаха средних значений скорости регрессивных саккад, что может коррелировать с качеством транссаккадического запоминания и рабочей памятью. Учитывая прямо пропорциональную связь между скоростью саккад и частотой разрядов пачечных нейронов центра генерации саккад, интегралом от этой частоты и амплитудой саккад (Leigh, Zee, 2015), а также взаимосвязь между амплитудой саккад и объемом рабочей памяти (Luke et al., 2018), можно предположить, что транссаккадическое запоминание в некоторой степени зависит от стабильности скорости саккад. В противном случае транссаккадический перенос информации также становится нестабильным. Тогда увеличение размаха значений скорости и амплитуды регрессивных саккад во всех группах кроме $\mathrm{R}$ можно рассматривать как признак относительной или минимальной дисрегуляции стволовых центров генерации саккад. Дисрегуляция между стволовыми центрами генерации саккад, нейрональными интеграторами и мозжечком может отражаться в увеличении размаха значений окуломоторных параметров (Sparks, 2002; Soetedjo et al., 2002; Voogd et al., 2012; Di Stasi et al., 2013; Leigh, Zee, 2015).

$\mathrm{y}$ школьников группы $\mathrm{W}$ имеется целостное, но неверное восприятие текста. С точки зрения теории лексической неоднозначности перед переходом к следующему 
слову ученик должен сделать выбор относительно значения текущего слова, но у школьников группы W выбор оказывается ошибочным, что сказывается на общем понимании смысла текста. Если наилучшее зрительное восприятие при чтении (интеграция фовеальной и парафовеальной информации) требует терминации (завершения) саккад в оптимальном для фиксации месте, то неточность программирования амплитуды саккад и, как следствие, увеличение доли дисметричных саккад (слишком большая или маленькая амплитуда саккад) ведут к увеличению времени фиксаций (Lopez-Perez et al., 2016). Вместе с тем известно, что фиксации после регрессивных саккад в большей степени способствуют разрешению контекстной неясности, чем лексической идентификации слова (Schotter et al., 2019). Исходя из этого, можно предположить, что увеличение длительности фиксации после регрессивных саккад в группе $\mathrm{W}$ в большей степени обусловлено недостаточно эффективной стратегией анализа информации или дефицитом антиципации (способность предвосхищать содержание текста), нежели состоянием функций памяти и/или внимания.

Дополняя вышеизложенное, следует упомянуть, что параметры движений глаз обладают высокой чувствительностью к самому широкому спектру экзо- и эндогенных факторов (Leigh, Zee, 2015; Vinuela-Navarro et al., 2017). Например, на продолжительность фиксаций может влиять наличие интроспекции во время чтения (Reichle et al., 2010), чтение вслух (Vorstius et al., 2014). На окуломоторную активность может повлиять изменение фоновой активности дефолтной системы, лежащей в основе работы других функциональных систем (Raichle, 2015). Кроме когнитивных факторов на окуломоторную активность при чтении в любом возрасте могут влиять факторы, тем или иным образом изменяющие общий тонус нервной ткани, например степень утомления, уровень кровоснабжения головного мозга и другие влияния со стороны вегетативной нервной системы.

\section{Заключение}

Для четырех групп учеников 7-8 лет с разным уровнем сформированности когнитивного компонента чтения определены особенности саккадической окуломоторной активности. Результаты данного исследования дают возможность более точной характеристики когнитивных функций при чтении. Полученные результаты могут оказаться полезными для оптимизации обучения чтению, внести вклад в фундаментальное понимание обеспечения когнитивных процессов головным мозгом. В заключение стоит отметить, что при дальнейшей работе по данной теме планируется увеличение размера выборки в группах NoA и RwP и привлечение метода ЭЭГ для уточнения различий функционирования нейрофизиологических систем между группами школьников с разным уровнем понимания читаемого.

\section{Благодарности / Acknowledgments}

Наш коллектив желает выразить свою благодарность Школе будущего и гимназии № 32 города Калининграда за помощь в наборе участников для исследования.

The authors are grateful to School of Future and School No. 32 in Kaliningrad for their assistance in selecting research participants. 


\section{Список литературы / References}

Безруких М.М., Иванов В.В. (2015) Окуломоторная активность при чтении у детей с разной степенью сформированностью навыка (Сообщение 2. Особенности окуломоторной активности у хорошо и плохо читающих детей 9-10 лет). Новые исследования, 43(2): 4-12 [Bezrukikh M.M., Ivanov V.V. (2015) Reading oculomotor activity in children with different reading skills (Message 2: peculiarities of oculomotor activity in 9-10-year-old children with good and poor reading skills). New Research [Novye issledovaniya], 43(2): 4-12]

Гарипова А.Б. (2018) Айтрекинг в методике обучения чтению на английском языке. Казанский лингвистический журнал, 1(2): 86-94 [Garipova A.B. (2018) Eye-tracking in teaching methods of reading in English. Kazan Linguistic Journal [Kazanskii lingvisticheskii zhurnal], 1(2): 86-94 (in Russian)]

Дубасова А.В. (2010) Движения глаз во время чтения: от общих теорий к частным. URL: https://www.academia.edu/3074458 [Dubasova A.V. (2010) Eye movements during reading: from general to specific theories. URL: https://www.academia.edu/3074458 (in Russian)]

Иванов В.В., Демидов А.А., Безруких М.М. (2010) Окуломоторная активность при чтении у детей с разной степенью сформированности навыка чтения. Экспериментальная психология в России, с. 611-616 [Ivanov V.V., Demidov A.A., Bezrukikh M.M. (2010) Oculomotor activity while reading in children with different reading skills. Experimental psychology in Russia, p. 611-616 (in Russian)]

Aminihajibashi S., Hagen T., Foldal M.D., Laeng B., Espeseth T. (2019) Individual differences in resting-state pupil size: Evidence for association between working memory capacity and pupil size variability. International Journal of Psychophysiology, 140: 1-7

Bezrukikh M.M., Ivanov V.V. (2013) Eye movements in the process of reading as an indicator of development of reading skill. Human Physiology, 39: 68-77

Clifton C., Ferreira F., Henderson J.M., Inhoff A.W., Liversedge S.P., Reichle E.D., Schotter E.R. (2016) Eye movements in reading and information processing: Keith Rayner's 40-year legacy. Journal of Memory and Language, 86: 1 - 19

Di Stasi L., Catena A., Canas J., Macknik S., Martinez-Conde S. (2013) Saccadic velocity as an arousal index in naturalistic task. Neuroscience and Biobehavioral Reviews, 37(5): 968-975

Eckstein M.K., Guerra-Carrillo B., Singley A.T.M., Bunge S.A. (2017) Beyond eye gaze: What else can eyetracking reveal about cognition and cognitive development. Developmental Cognitive Neuroscience, 25: 69-91

Edwards G., VanRullen R., Cavanagh P. (2018) Decoding trans-saccadic memory. Journal of Neuroscience, 38(5): 1114-1123

Foster T.E., Ardoin S.P., Binder K.S. (2018) Reliability and validity of eye movement measures of children's reading. Reading Research Quarterly, 53(1): 71-89

Frey A., Ionescu G., Lemaire B., López-Orozco F., Baccino T., Guérin-Dugué A. (2013) Decisionmaking in information seeking on texts: an eye-fixation-related potentials investigation. Frontiers in Systems Neuroscience, 7: 39

Hakim N., Adam K.C.S., Gunseli E., Awh E., Vogel E.K. (2019) Dissecting the neural focus of attention reveals distinct processes for spatial attention and object-based storage in visual working memory. Psychological Science, 30(4): 526-540 
Hutton S.B. (2008) Cognitive control of saccadic eye movements. Brain and Cognition, 68(3): 327-340

Irwin D.E., Thomas L.E. (2007) The effect of saccades on number processing. Perception \& Psychophysics, 69(3): $450-458$

Keil V., Hepach R., Vierrath S., Caffier D., Tuschen-Caffier B., Klein C., Schmitz J. (2018) Children with social anxiety disorder show blunted pupillary reactivity and altered eye contact processing in response to emotional faces: Insights from pupillometry and eye movements. Journal of Anxiety Disorders, 58: 61-69

Kustov A.A., Robinson D.L. (1996) Shared neural control of attentional shifts and eye movements. Nature, 384(6604): 74-77

Leigh R.J., Zee D.S. (2015) The neurology of eye movements. Oxford University Press

Lopez-Perez P.J., Dampure J., Hernández-Cabrera J.A., Barber H.A. (2016) Semantic parafovealon-foveal effects and preview benefits in reading: Evidence from Fixation Related Potentials. Brain and Language, 162: $29-34$

Luke S.G., Darowski E.S., Gale S.D. (2018) Predicting eye-movement characteristics across multiple tasks from working memory and executive control. Memory \& Cognition, 46(5): 826-839

Mäki-Marttunen V., Hagen T., Espeseth T. (2019) Task context load induces reactive cognitive control: An fMRI study on cortical and brain stem activity. Cognitive, Affective, \& Behavioral Neuroscience, 19(4): 945-965

Mather M., Harley C.W. (2016) The locus coeruleus: Essential for maintaining cognitive function and the aging brain. Trends in Cognitive Sciences, 20(3): 214-226

Optican L.M., Pretegiani E. (2017) What stops a saccade? Philosophical Transactions of the Royal Society B: Biological Sciences, 372(1718): 20160194

Raichle M.E. (2015) The brain's default mode network. Annual Review of Neuroscience, Vol. 38, p. $433-447$

Reichle E.D., Reineberg A.E., Schooler J.W. (2010) Eye movements during mindless reading. Psychological Science, 21(9): 1300-1310

Rayner K., Reingold E.M. (2015) Evidence for direct cognitive control of fixation durations during reading. Current Opinion in Behavioral Sciences, 1: 107 - 112

Rodrigues A.P., Rebola J., Pereira M., van Asselen M., Castelo-Branco M. (2019) Neural responses of the anterior ventral occipitotemporal cortex in developmental dyslexia: beyond the visual word form area. Investigative Ophthalmology \& Visual Science, 60(4): 1063-1068

Rucci M., Poletti M. (2015) Control and functions of fixational eye movements. Annual Review of Vision Science, 1: 499-518

Schmahmann J.D., Guell X., Stoodley C.J., Halko M.A. (2019) The theory and neuroscience of cerebellar cognition. Annual Review of Neuroscience, 42: 337-364

Schneegans S., Spencer J.P., Schöner G., Hwang S., Hollingworth A. (2014) Dynamic interactions between visual working memory and saccade target selection. Journal of Vision, 14(11): 9

Schotter E.R., Li C., Gollan T.H. (2019) What reading aloud reveals about speaking: Regressive saccades implicate a failure to monitor, not inattention, in the prevalence of intrusion errors on function words. Quarterly Journal of Experimental Psychology, 72(8): 2032-2045

Seassau M., Bucci M.P. (2013) Reading and visual search: a developmental study in normal children. PLoS One, 8(7): e70261 
Sharp D.J., Scott S.K., Wise R.J.S. (2004) Monitoring and the controlled processing of meaning: distinct prefrontal systems. Cerebral Cortex, 14(1): 1 - 10

Smadja U., Grusky M., Artzi Y., Naaman M. (2019) Understanding reader backtracking behavior in online news articles. The Web Conference 2019 - Proceedings of the World Wide Web Conference, $W W W$ 2019. p. 3237-3243

Soetedjo R., Kaneko C.R.S., Fuchs A.F. (2002) Evidence that the superior colliculus participates in the feedback control of saccadic eye movements. Journal of Neurophysiology, 87(2): $679-695$

Sparks D. (2002) The brainstem control of saccadic eye movements. Nature Reviews Neuroscience, 3(12): $952-964$

Steindorf L., Rummel J. (2020) Do your eyes give you away? A validation study of eye-movement measures used as indicators for mindless reading. Behavior Research Methods, 52(1): 162-176

Stewart E.E.M., Schütz A.C. (2018) Attention modulates trans-saccadic integration. Vision Research, 142: 1-10

Vinuela-Navarro V., Erichsen J.T., Williams C., Woodhouse J.M. (2017) Saccades and fixations in children with delayed reading skills. Ophthalmic and Physiological Optics, 37(4): 531-541

Voogd J., Schraa-Tam C.K.L., van der Geest J.N., De Zeeuw C.I. (2012) Visuomotor cerebellum in human and nonhuman primates. Cerebellum, 11(2): 392-410

Vorstius C., Radach R., Lonigan C.J. (2014) Eye movements in developing readers: A comparison of silent and oral sentence reading. Visual Cognition, 22(3-4): 458-485

Yeh Y.C., Tsai J.L., Hsu W.C., Lin C.F. (2014) A model of how working memory capacity influences insight problem solving in situations with multiple visual representations: An eye tracking analysis. Thinking Skills and Creativity, 13: 153-167

Zhao M., Gersch T.M., Schnitzer B.S., Dosher B.A., Kowler E. (2012) Eye movements and attention: The role of pre-saccadic shifts of attention in perception, memory and the control of saccades. Vision Research, 74: 40-60 